Bull. Korean Math. Soc. 49 (2012), No. 1, pp. 213-222

http://dx.doi.org/10.4134/BKMS.2012.49.1.213

\title{
WHICH K3 SURFACES WITH PICARD NUMBER 19 COVER AN ENRIQUES SURFACE
}

\author{
KWANGWOO LEE
}

Abstract. We determine which K3 surface with Picard number 19 is a K3 cover of an Enriques surface.

\section{Introduction}

Let $X$ be a K3 surface with Picard number, $\rho(X), 19$ over the field $\mathbb{C} . T_{X}$ can be denoted by the following intersection matrix

$$
T_{X}=\left(\begin{array}{ccc}
2 a & d & e \\
d & 2 b & f \\
e & f & 2 c
\end{array}\right)
$$

with respect to a basis $\{x, y, z\}$. Since the transcendental lattice $T_{X}$ of $X$ has signature $(2,1)$, without loss of generality, we may assume that $z^{2}=2 c<0$. Let $U$ and $E_{8}$ denote the even unimodular lattices of signature $(1,1)$ and $(0,8)$ respectively. Keum showed that every algebraic Kummer surface is the K3 cover of some Enriques surface in [3] with the following criterion.

Theorem 1.1 (Keum, [3]). (Criterion for a K3 surface to cover an Enriques surface) Let $X$ be an algebraic K3 surface. Assume that $l\left(T_{X}\right)+2 \leq \rho(X)$, where $l\left(T_{X}\right)$ is the length of $T_{X}$ (This is true if $\rho(X) \geq 12$ ). Then, the following are equivalent.

(i) $X$ admits a fixed-point-free involution.

(ii) There exists a primitive embedding of $T_{X}$ into $\Lambda^{-}=U \oplus U(2) \oplus E_{8}(2)$ such that the orthogonal complement of $T_{X}$ in $\Lambda^{-}$contains no vectors of selfintersection -2 .

Following the work of Keum, Ali Sinan Sertöz determined the necessary and sufficient conditions for a singular K3 surface $(\rho(X)=20)$ to cover an Enriques surface, [5]. He used the following lemma to show that a given lattice embedding is a primitive.

Received October 20, 2010; Revised March 19, 2011.

2010 Mathematics Subject Classification. 14J28.

Key words and phrases. K3 surface, Picard number, lattice embedding.

(C)2012 The Korean Mathematical Society 
Lemma $1.2([5])$. A lattice embedding is primitive if and only if the greatest common divisor of the maximal minors of the embedding matrix with respect to any choice of basis is 1 .

In this paper we will use these for the case $\rho(X)=19$. For the definitions and basic facts about K3 surfaces we refer to [1].

Our purpose is to show following theorems:

Theorem 1.3. If $X$ is a K3 surface with a transcendental lattice given as in (1), then the K3 surface satisfying one of the following conditions is a K3 cover of an Enriques surface.

1. $a, b, c$, and def are even.

2. $a$ is odd; $b$ and $c$ are even $\left\{\begin{array}{l}\text { (i) de } f \text { is odd. } \\ \text { (ii) } f \text { is even and } d \text { is odd } \\ \text { or } f \text { is even and } e \text { is odd. }\end{array}\right.$

3. $b$ is odd; $a$ and $c$ are even $\left\{\begin{array}{l}\text { (i) } e \text { is even and } d \text { is odd } \\ \text { or } e \text { is even and } f \text { is odd. } \\ \text { (ii) def is odd. }\end{array}\right.$

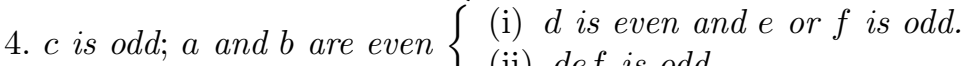

5. Only $a$ and $f$ are even.

6. Only $b$ and $e$ are even.

7. Only $c$ and $d$ are even.

Theorem 1.4. If $X$ is a K3 surface with a transcendental lattice given as in (1), then the K3 surface satisfying one of the following conditions is not a K3 cover of any Enriques surface.

1. $a, b$, and $c$ are even; def is odd.

2. af is odd; $b, c$, and de are even.

3. be is odd; a,c, and $d f$ are even.

4. $c d$ is odd; $a, b$, and ef are even.

5. $a$ is even and bc is odd $\{$ (i) $f$ is odd.

(ii) $f$ is even and $d+e$ is odd.

6. $b$ is even and ac is odd

(i) $e$ is odd.

7. $c$ is even and $a b$ is odd \{

(ii) $e$ is even and $d+f$ are odd.

(i) $d$ is odd.

8. abc is odd; $d$, e, or $f$ is odd.

(ii) $d$ is even and $e+f$ is odd.

Remark 1.5. The remaining cases are as follows:

1. Only $a$ is odd.

2. Only $b$ is odd.

3. Only $c$ is odd.

4. Only $a$ and $b$ are odd.

5. Only $b$ and $c$ are odd.

6. Only $a$ and $c$ are odd. 
7. Only $a, b$, and $c$ are odd.

However, since we will show that these are all equivalent, it is sufficient to consider only one of these.

Before we proceed to the proof of the theorems we will also need the following lemma. We recall that for $A \in S L_{3}(\mathbb{Z}), T_{X}^{\prime}=A T_{X} A^{\operatorname{tr}}=\left(\begin{array}{ccc}2 a^{\prime} & d^{\prime} & e^{\prime} \\ d^{\prime} & 2 b^{\prime} & f^{\prime} \\ e^{\prime} & f^{\prime} & 2 c^{\prime}\end{array}\right)$ is $\mathbb{Z}-$ equivalent to $T_{X}$.

Lemma 1.6. Let $T_{X}$ be a lattice given as in (1) with $c<0$. Then, $T_{X}$ is $\mathbb{Z}$-equivalent to $T_{X}^{\prime}$ with $b^{\prime}, c^{\prime}<0$ and $a^{\prime}=a$.

Proof. Assume that $b \geq 0$ (If $b<0$, there is nothing to prove). Let $A=\left(\begin{array}{lll}1 & 0 & 0 \\ 0 & 1 & \alpha \\ 0 & 0 & 1\end{array}\right)$, where $\alpha \in \mathbb{Z}$. Since $A \in S L_{3}(\mathbb{Z})$, the matrix $A T_{X} A^{\text {tr }}$ is $\mathbb{Z}$-equivalent to $T_{X}$. Now $T_{X}^{\prime}=A T_{X} A^{t r}=\left(\begin{array}{ccc}2 a^{\prime} & d^{\prime} & e^{\prime} \\ d^{\prime} & 2 b^{\prime} & f^{\prime} \\ e^{\prime} & f^{\prime} & 2 c^{\prime}\end{array}\right)$, where $a^{\prime}=a, b^{\prime}=b+\alpha^{2} c+\alpha f, c^{\prime}=c$, $d^{\prime}=d+\alpha e, e^{\prime}=e, f^{\prime}=2 \alpha c+f$.

Case 1. $f \neq 0$.

Let $\alpha=-n f$, where $n \in \mathbb{Z}^{+}$. Then $b^{\prime}=b+(-n f)^{2} c+(-n) f^{2}<0$ for some $n$. Case 2. $f=0$.

We can take $\alpha=n \in \mathbb{Z}$ such that $b^{\prime}<0$, where $b^{\prime}=b+n^{2} c$.

Case 3. $b=0$.

If $f \leq 0$, let $\alpha=1$. Then $b^{\prime}=c+f<0$. If $f>0$, let $\alpha=-1$. Then $b^{\prime}=c-f<0$.

From now on, we assume that $b$ and $c$ in $T_{X}$ of (1) are negative.

Theorem 1.7 (Nikulin, [4]). A primitive embedding of an even non-degenerate lattice $L$ of signature $\left(s_{+}, s_{-}\right)$into an even unimodular lattice $M$ of signature $\left(t_{+}, t_{-}\right)$exists provided that

$$
s_{+} \leq t_{+}, s_{-} \leq t_{-}, \text {and } l(L)+1 \leq \operatorname{rank}(M)-\operatorname{rank}(L),
$$

where $l(L)$ is the length of $L$. Furthermore, if the three inequalities are all strict, then the primitive embedding is unique.

The following corollary will be used later.

Corollary 1.8. There is a primitive embedding of $\langle-2 m\rangle$ into $E_{8}$ for any positive integer $m$.

\section{Proof of Theorem 1.3}

Let $\{x, y, z\}$ be a basis of the transcendental lattice $T_{X}$ and let $\left\{u_{1}, u_{2}\right\}$ and $\left\{v_{1}, v_{2}\right\}$ be the standard bases of $U$ and $U(2)$, respectively. We prove Theorem 1.3 by showing the existence of primitive embedding of $T_{X}$ into $\Lambda^{-}$such that the orthogonal complement of $\operatorname{Im} \phi$ contains no $(-2)$-vectors. 
1-i) $a, b, c$, and $e$ are even.

Consider the mapping $\phi: T_{X} \longrightarrow \Lambda^{-}$defined as

$$
\begin{aligned}
& \phi(x)=d u_{2}+v_{1}+\frac{a}{2} v_{2}, \\
& \phi(y)=u_{1}+b u_{2}, \\
& \phi(z)=f u_{2}+\frac{e}{2} v_{2}+w,
\end{aligned}
$$

where by Corollary 1.8 we can choose a primitive element $w$ of $E_{8}(2)$ with $w^{2}=2 c, c<0$. Then $\phi$ is an embedding and by Lemma $1.2, \phi$ is a primitive embedding. Assume that $s=x_{1} u_{1}+x_{2} u_{2}+x_{3} v_{1}+x_{4} v_{2}+w^{\prime}$ is an element of orthogonal complement of the $\operatorname{Im} \phi$. Then $s \cdot \phi(y)=0$ induces $b x_{1}+x_{2}=0$. Since $b$ is even, $x_{2}$ is even. Thus $s \cdot s=2 x_{1} x_{2}+4 x_{3} x_{4}+w^{\prime 2} \equiv 0(\bmod 4)$ and hence cannot be -2 .

1-ii) $a, b, c$, and $f$ are even.

Consider the mapping $\phi: T_{X} \longrightarrow \Lambda^{-}$defined as

$$
\begin{aligned}
& \phi(x)=u_{1}+a u_{2}, \\
& \phi(y)=d u_{2}+v_{1}+\frac{b}{2} v_{2}, \\
& \phi(z)=e u_{2}+\frac{f}{2} v_{2}+w,
\end{aligned}
$$

where by Corollary 1.8 we can choose a primitive element $w$ of $E_{8}(2)$ with $w^{2}=2 c, c<0$. This is an embedding and by Lemma 1.2, it is primitive. Assume that $s=x_{1} u_{1}+x_{2} u_{2}+x_{3} v_{1}+x_{4} v_{2}+w^{\prime}$ is an element of orthogonal complement of the $\operatorname{Im} \phi$. Then $s \cdot \phi(x)=0$ induces $a x_{1}+x_{2}=0$. Since $a$ is even, $x_{2}$ is even. Thus $s \cdot s \equiv 0(\bmod 4)$ and hence cannot be -2 .

1-iii) $a, b, c$, and $d$ are even ( $e f$ is odd, otherwise 1-i) or 1-ii)).

We use the base change by $\left(\begin{array}{lll}1 & 1 & 0 \\ 0 & 1 & 0 \\ 0 & 0 & 1\end{array}\right)$, where $a^{\prime}=a+b+d, b^{\prime}=b, c^{\prime}=c$, $d^{\prime}=2 b+d, e^{\prime}=e+f$, and $f^{\prime}=f$. Then $a^{\prime}, b^{\prime}, c^{\prime}$, and $e^{\prime}$ are even. Thus this case is reduced to Case 1-i).

2-i) Only $b$ and $c$ are even.

We use the base change by $\left(\begin{array}{lll}1 & 1 & 0 \\ 0 & 1 & 0 \\ 0 & 0 & 1\end{array}\right)$, where $a^{\prime}=a+b+d, b^{\prime}=b, c^{\prime}=c$, $d^{\prime}=2 b+d, e^{\prime}=e+f$, and $f^{\prime}=f$. Then $a^{\prime}, b^{\prime}, c^{\prime}$, and $e^{\prime}$ are even. Thus this case is reduced to Case 1.

2-ii) $b, c$, and $f$ are even, $a$ is odd, and either $d$ or $e$ is odd.

If $d$ is odd, then we use the base change by $\left(\begin{array}{lll}1 & 1 & 0 \\ 0 & 1 & 0 \\ 0 & 0 & 1\end{array}\right)$, where $a^{\prime}=a+b+d$, $b^{\prime}=b, c^{\prime}=c, d^{\prime}=2 b+d, e^{\prime}=e+f$, and $f^{\prime}=f$. Then $a^{\prime}, b^{\prime}, c^{\prime}$, and $f^{\prime}$ are even. Thus this case is reduced to Case 1 .

If $e$ is odd, then we use the base change by $\left(\begin{array}{lll}1 & 0 & 1 \\ 0 & 1 & 0 \\ 0 & 0 & 1\end{array}\right)$, where $a^{\prime}=a+c+e$, $b^{\prime}=b, c^{\prime}=c, d^{\prime}=d+f, e^{\prime}=2 c+e$, and $f^{\prime}=f$. Then $a^{\prime}, b^{\prime}, c^{\prime}$, and $f^{\prime}$ are even. Thus this case is reduced to Case 1. 
3-i) $a, c$, and $e$ are even, $b$ is odd, and either $d$ or $f$ is odd.

Consider the mapping $\phi: T_{X} \longrightarrow \Lambda^{-}$defined as

$$
\begin{aligned}
& \phi(x)=d u_{2}+v_{1}+\frac{a}{2} v_{2}, \\
& \phi(y)=u_{1}+b u_{2}, \\
& \phi(z)=f u_{2}+\frac{e}{2} v_{2}+w,
\end{aligned}
$$

where by Corollary 1.8 we can choose a primitive element $w$ of $E_{8}(2)$ with $w^{2}=$ $2 c, c<0$. This is an embedding and by Lemma 1.2, it is primitive. Assume that $s=x_{1} u_{1}+x_{2} u_{2}+x_{3} v_{1}+x_{4} v_{2}+w^{\prime}$ is an element of orthogonal complement of the $\operatorname{Im} \phi$. Then $s \cdot \phi(x)=d x_{1}+a x_{3}+2 x_{4}=0, s \cdot \phi(z)=f x_{1}+e x_{3}+w w^{\prime}=0$. Since $d$ or $f$ is odd, $x_{1}$ is even. Thus $s \cdot s \equiv 0(\bmod 4)$ and hence cannot be -2 .

3-ii) Only $a$ and $c$ are even.

We use the base change by $\left(\begin{array}{lll}1 & 1 & 1 \\ 0 & 1 & 0 \\ 0 & 0 & 1\end{array}\right)$, where $a^{\prime}=a+b+c+d+e+f, b^{\prime}=b$, $c^{\prime}=c, d^{\prime}=2 b+d+f, e^{\prime}=2 c+e+f$, and $f^{\prime}=f$. Then this case is reduced to Case 3-i).

4 -i) $a, b$, and $d$ are even, $c$ is odd, and either $e$ or $f$ is odd.

Consider the mapping $\phi: T_{X} \longrightarrow \Lambda^{-}$defined as

$$
\begin{aligned}
& \phi(x)=e u_{2}+v_{1}+\frac{a}{2} v_{2}, \\
& \phi(y)=f u_{2}+\frac{d}{2} v_{2}+w, \\
& \phi(z)=u_{1}+c u_{2},
\end{aligned}
$$

where by Corollary 1.8 we can choose a primitive element $w$ of $E_{8}(2)$ with $w^{2}=$ $2 b, b<0$. This is an embedding and by Lemma 1.2 , it is primitive. Assume that $s=x_{1} u_{1}+x_{2} u_{2}+x_{3} v_{1}+x_{4} v_{2}+w^{\prime}$ is an element of orthogonal complement of the $\operatorname{Im} \phi$. Then $s \cdot \phi(x)=e x_{1}+a x_{3}+2 x_{4}=0, s \cdot \phi(y)=f x_{1}+d x_{3}+w w^{\prime}=0$. Since $e$ or $f$ is odd, $x_{1}$ is even. Thus $s \cdot s \equiv 0(\bmod 4)$ and hence cannot be -2 .

4-ii) Only $a$ and $b$ are even.

We use the base change by $\left(\begin{array}{lll}1 & 0 & 1 \\ 0 & 1 & 0 \\ 0 & 0 & 1\end{array}\right)$, where $a^{\prime}=a+c+e, b^{\prime}=b, c^{\prime}=c$, $d^{\prime}=d+f, e^{\prime}=2 c+e$, and $f^{\prime}=f$. Then this case is reduced to Case 4-i).

5) Only $a$ and $f$ are even.

Since we assume that $b, c<0$, we split into two cases.

i) $f \geq 0$.

We use the base change by $\left(\begin{array}{ccc}1 & 0 & 0 \\ 0 & 1 & -1 \\ 0 & 0 & 1\end{array}\right)$, where $a^{\prime}=a, b^{\prime}=b+c-f, c^{\prime}=c$, $d^{\prime}=d-e, e^{\prime}=e$, and $f^{\prime}=-2 c+f$. Then this case is reduced to Case 4-i).

ii) $f<0$.

We use the base change by $\left(\begin{array}{lll}1 & 0 & 0 \\ 0 & 1 & 1 \\ 0 & 0 & 1\end{array}\right)$, where $a^{\prime}=a, b^{\prime}=b+c+f, c^{\prime}=c$, $d^{\prime}=d+e, e^{\prime}=e$, and $f^{\prime}=2 c+f$. Then this case is reduced to Case $\left.4-\mathrm{i}\right)$. 
6) Only $b$ and $e$ are even.

We use the base change by $\left(\begin{array}{lll}1 & 1 & 0 \\ 0 & 1 & 0 \\ 0 & 0 & 1\end{array}\right)$, where $a^{\prime}=a+b+d, b^{\prime}=b, c^{\prime}=c$, $d^{\prime}=2 b+d, e^{\prime}=e+f$, and $f^{\prime}=f$. Then this case is reduced to Case 4-ii).

7) Only $c$ and $d$ are even.

Since we assume that $b, c<0$, we split into two cases.

i) $f \geq 0$.

We use the base change by $\left(\begin{array}{ccc}1 & 0 & 1 \\ 0 & 1 & -1 \\ 0 & 0 & 1\end{array}\right)$, where $a^{\prime}=a+c+e, b^{\prime}=b+c-f$, $c^{\prime}=c, d^{\prime}=-2 c+d-e+f, e^{\prime}=2 c+e$, and $f^{\prime}=-2 c+f$. Then $a^{\prime}, b^{\prime}, c^{\prime}$, and $d^{\prime}$ are even. Thus this case is reduced to Case 1 .

ii) $f<0$.

We use the base change by $\left(\begin{array}{lll}1 & 0 & 1 \\ 0 & 1 & 1 \\ 0 & 0 & 1\end{array}\right)$, where $a^{\prime}=a+c+e, b^{\prime}=b+c+f, c^{\prime}=c$, $d^{\prime}=2 c+d+e+f, e^{\prime}=2 c+e$, and $f^{\prime}=2 c+f$. Then $a^{\prime}, b^{\prime}, c^{\prime}$, and $d^{\prime}$ are even. Thus this case is reduced to Case 1 .

\section{Proof of Theorem 1.4}

Let $\{x, y, z\}$ be a basis of the transcendental lattice $T_{X}$ and let $\left\{u_{1}, u_{2}\right\}$ and $\left\{v_{1}, v_{2}\right\}$ be the standard bases of $U$ and $U(2)$, respectively. We derive a contradiction if an embedding of $T_{X}$ into $\Lambda^{-}$exists.

1) $a, b$, and $c$ are even; def is odd.

Consider the mapping $\phi: T_{X} \longrightarrow \Lambda^{-}$defined generically as

$$
\left\{\begin{array}{l}
\phi(x)=a_{1} u_{1}+a_{2} u_{2}+a_{3} v_{1}+a_{4} v_{2}+w_{1}, \\
\phi(y)=b_{1} u_{1}+b_{2} u_{2}+b_{3} v_{1}+b_{4} v_{2}+w_{2}, \\
\phi(z)=c_{1} u_{1}+c_{2} u_{2}+c_{3} v_{1}+c_{4} v_{2}+w_{3},
\end{array}\right.
$$

where the $a_{i}$ 's, $b_{i}$ 's, and $c_{i}$ 's are integers, $w_{i} \in E_{8}(2)$. Assume that $\phi$ is an embedding, i.e.

$$
\left\{\begin{array}{l}
\phi(x) \cdot \phi(x)=2 a_{1} a_{2}+4 a_{3} a_{4}+w_{1}^{2}=2 a \\
\phi(y) \cdot \phi(y)=2 b_{1} b_{2}+4 b_{3} b_{4}+w_{2}^{2}=2 b \\
\phi(z) \cdot \phi(z)=2 c_{1} c_{2}+4 c_{3} c_{4}+w_{3}^{2}=2 c \\
\phi(x) \cdot \phi(y)=a_{1} b_{2}+a_{2} b_{1}+2 a_{3} b_{4}+2 a_{4} b_{3}+w_{1} w_{2}=d \\
\phi(x) \cdot \phi(z)=a_{1} c_{2}+a_{2} c_{1}+2 a_{3} c_{4}+2 a_{4} c_{3}+w_{1} w_{3}=e \\
\phi(y) \cdot \phi(z)=b_{1} c_{2}+b_{2} c_{1}+2 b_{3} c_{4}+2 b_{4} c_{3}+w_{2} w_{3}=f .
\end{array}\right.
$$

Since $a$ is even and $d$ is odd, either $a_{1}$ or $a_{2}$ is even; similarly for $b_{1}, b_{2}$ and $c_{1}, c_{2}$. Without loss of generality, we may assume that $a_{1}$ is even. Then since $d$ and $e$ are odd $a_{2}, b_{1}$, and $c_{1}$ are odd. Hence $b_{2}$ and $c_{2}$ are even. Then $f$ is even which is a contradiction, so $T_{X}$ has no embedding into $\Lambda^{-}$.

2) af is odd; $b, c$, and de are even.

Consider the mapping $\phi: T_{X} \longrightarrow \Lambda^{-}$defined generically as in (2). Assume that $\phi$ is an embedding. Since $a$ is odd and $b, c$ are even, from (3) $a_{1}, a_{2}$ are odd, $b_{1}$ or $b_{2}$ is even, and $c_{1}$ or $c_{2}$ is even. Also, since $f$ is odd, $\left(\begin{array}{c}b_{1} \text { and } c_{2} \text { are even } \\ b_{2} \text { and } c_{1} \text { are odd }\end{array}\right)$ or 
$\left(\begin{array}{c}b_{2} \text { and } c_{1} \text { are even } \\ b_{1} \text { and } c_{2} \text { are odd }\end{array}\right)$. Since $d$ or $e$ is even, $b_{1}, b_{2}$ are even or $c_{1}, c_{2}$ are even. So $f$ is even. Hence, $T_{X}$ has no embedding into $\Lambda^{-}$.

3) be is odd; $a, c$, and $d f$ are even.

Consider the mapping $\phi: T_{X} \longrightarrow \Lambda^{-}$defined generically as in (2). Assume that $\phi$ is an embedding. Since $b$ and $e$ are odd, from (3) $b_{1}, b_{2}, a_{1} c_{2}+a_{2} c_{1}$ are odd. Also, since $a, c$ are even, $\left(\begin{array}{l}a_{1} \text { and } c_{2} \text { are even } \\ a_{2} \text { and } c_{1} \text { are odd }\end{array}\right)$ or $\left(\begin{array}{c}a_{2} \text { and } c_{1} \text { are even } \\ a_{1} \text { and } c_{2} \text { are odd }\end{array}\right)$. Then, both $d$ and $f$ are odd. Hence, $T_{X}$ has no embedding into $\Lambda^{-}$.

4) $c d$ is odd; $a, b$, and $e f$ are even.

Consider the mapping $\phi: T_{X} \longrightarrow \Lambda^{-}$defined generically as in (2). Assume that $\phi$ is an embedding. Since $c$ and $d$ are odd, from (3) $c_{1}, c_{2}, a_{1} b_{2}+a_{2} b_{1}$ are odd. Also, since $a, b$ are even, $\left(\begin{array}{l}a_{1} \text { and } b_{2} \text { are even } \\ a_{2} \text { and } b_{1} \text { are odd }\end{array}\right)$ or $\left(\begin{array}{c}a_{2} \text { and } b_{1} \text { are even } \\ a_{1} \text { and } b_{2} \text { are odd }\end{array}\right)$. Then, both $e$ and $f$ are odd. Hence, $T_{X}$ has no embedding into $\Lambda^{-}$.

5 -i) $a$ is even and $b c f$ is odd.

Consider the mapping $\phi: T_{X} \longrightarrow \Lambda^{-}$defined generically as in (2). Assume that $\phi$ is an embedding. Since $b$ and $c$ are odd, from $(3) b_{1}, b_{2}, c_{1}, c_{2}$ are odd. Then, $f$ is even. Hence, $T_{X}$ has no embedding into $\Lambda^{-}$.

5-ii) $a$ and $f$ are even; $b, c$, and $d+e$ are odd.

Consider the mapping $\phi: T_{X} \longrightarrow \Lambda^{-}$defined generically as in (2). Assume that $\phi$ is an embedding. Without loss of generality, we may assume that $d$ is odd. Then, either $a_{1}$ or $a_{2}$ is even. Since $b_{1}, b_{2}, c_{1}$, and $c_{2}$ are odd, $e$ is also odd. That is, $d$ and $e$ have the same sign. Hence, $T_{X}$ has no embedding into $\Lambda^{-}$.

6 -i) $b$ is even and ace is odd.

Consider the mapping $\phi: T_{X} \longrightarrow \Lambda^{-}$defined generically as in (2). Assume that $\phi$ is an embedding. Since $a$ and $c$ are odd, from (3) $a_{1}, a_{2}, c_{1}$, and $c_{2}$ are odd. Then, $e$ is even. Hence, $T_{X}$ has no embedding into $\Lambda^{-}$.

6-ii) $b$ and $e$ are even; $a, c$, and $d+f$ are odd.

Consider the mapping $\phi: T_{X} \longrightarrow \Lambda^{-}$defined generically as in (2). Assume that $\phi$ is an embedding. Without loss of generality, we may assume that $d$ is odd. Then, either $b_{1}$ or $b_{2}$ is even. Since $a_{1}, a_{2}, c_{1}$, and $c_{2}$ are odd, $f$ is also odd. That is, $d$ and $f$ have the same parity. Hence, $T_{X}$ has no embedding into $\Lambda^{-}$.

$7-\mathrm{i}) c$ is even and $a b d$ is odd.

Consider the mapping $\phi: T_{X} \longrightarrow \Lambda^{-}$defined generically as in (2). Assume that $\phi$ is an embedding. Since $a$ and $b$ are odd, from (3) $a_{1}, a_{2}, b_{1}$, and $b_{2}$ are odd. Then, $d$ is even. Hence, $T_{X}$ has no embedding into $\Lambda^{-}$.

7-ii) $c$ and $d$ are even; $a, b$, and $e+f$ are odd.

Consider the mapping $\phi: T_{X} \longrightarrow \Lambda^{-}$defined generically as in (2). Assume that $\phi$ is an embedding. Without loss of generality, we may assume that $e$ is odd. Then, either $c_{1}$ or $c_{2}$ is even. Since $a_{1}, a_{2}, b_{1}$, and $b_{2}$ are odd, $f$ is also odd. That is, $e$ and $f$ have the same parity. Hence, $T_{X}$ has no embedding into $\Lambda^{-}$. 
8) $a, b$, and $c$ are odd; $d, e$, or $f$ is odd.

Consider the mapping $\phi: T_{X} \longrightarrow \Lambda^{-}$defined generically as in (2). Assume that $\phi$ is an embedding. Since $a, b$, and $c$ are odd, from (3) $a_{1}, a_{2}, b_{1}, b_{2}, c_{1}$, and $c_{2}$ are odd. Then, $d, e$, and $f$ are even. Hence, $T_{X}$ has no embedding into $\Lambda^{-}$.

\section{Remaining cases}

The remaining cases are as follows:

1. Only $a$ is odd.

2. Only $b$ is odd.

3. Only $c$ is odd.

4. Only $a$ and $b$ are odd.

5. Only $b$ and $c$ are odd.

6. Only $a$ and $c$ are odd.

7. Only $a, b$, and $c$ are odd.

However, these are all equivalent. First, we show that Cases 4 and 7 are equivalent to Case 2 and that Cases 5 and 6 are equivalent to Case 3.

Lemma 4.1. The case in which only $a$ and $b$ are odd is equivalent to the case in which only $b$ is odd.

Proof. We use the base change by $\left(\begin{array}{lll}1 & 1 & 1 \\ 0 & 1 & 0 \\ 0 & 0 & 1\end{array}\right)$, where $a^{\prime}=a+b+c+d+e+f$, $b^{\prime}=b, c^{\prime}=c, d^{\prime}=2 b+d+f, e^{\prime}=2 c+e+f$, and $f^{\prime}=f$. Then this case is reduced to the case in which only $b$ is odd.

Lemma 4.2. The case in which only $a, b$, and $c$ are odd is equivalent to the case in which only $b$ is odd.

Proof. Since we assume that $b, c<0$, we split into two cases.

i) $f \geq 0$.

We use the base change by $\left(\begin{array}{ccc}1 & 2 & 1 \\ 0 & 1 & 0 \\ 0 & -1 & 1\end{array}\right)$, where $a^{\prime}=a+4 b+c+2 d+e+2 f, b^{\prime}=b$, $c^{\prime}=b+c-f, d^{\prime}=4 b+d+f, e^{\prime}=-4 b+2 c-d+e+f$, and $f^{\prime}=-2 b+f$. Then this case is reduced to the case in which only $b$ is odd.

ii) $f<0$.

We use the base change by $\left(\begin{array}{lll}1 & 2 & 1 \\ 0 & 1 & 0 \\ 0 & 1 & 1\end{array}\right)$, where $a^{\prime}=a+4 b+c+2 d+e+2 f, b^{\prime}=b$, $c^{\prime}=b+c+f, d^{\prime}=4 b+d+f, e^{\prime}=4 b+2 c+d+e+3 f$, and $f^{\prime}=2 b+f$. Then this case is reduced to the case in which only $b$ is odd.

Lemma 4.3. The case in which only $b$ and $c$ are odd is equivalent to the case in which only $c$ is odd.

Proof. Since we assume that $b, c<0$, we split into two cases.

i) $f \geq 0$.

We use the base change by $\left(\begin{array}{ccc}1 & 0 & 0 \\ 0 & 1 & -1 \\ 0 & 0 & 1\end{array}\right)$, where $a^{\prime}=a, b^{\prime}=b+c-f, c^{\prime}=c$, 
$d^{\prime}=d-e, e^{\prime}=e$, and $f^{\prime}=-2 c+f$. Then this case is reduced to the case in which only $c$ is odd.

ii) $f<0$.

We use the base change by $\left(\begin{array}{lll}1 & 0 & 0 \\ 0 & 1 & 1 \\ 0 & 0 & 1\end{array}\right)$, where $a^{\prime}=a, b^{\prime}=b+c+f, c^{\prime}=c$, $d^{\prime}=d+e, e^{\prime}=e$, and $f^{\prime}=2 c+f$. Then this case is reduced to the case in which only $c$ is odd.

Lemma 4.4. The case in which only $a$ and $c$ are odd is equivalent to the case in which only $c$ is odd.

Proof. We use the base change by $\left(\begin{array}{lll}1 & 0 & 1 \\ 0 & 1 & 0 \\ 0 & 0 & 1\end{array}\right)$, where $a^{\prime}=a+c+e, b^{\prime}=b, c^{\prime}=c$, $d^{\prime}=d+f, e^{\prime}=2 c+e$, and $f^{\prime}=f$. Then this case is reduced to the case in which only $c$ is odd.

Now in order to show that Cases 1,2 , and 3 are equivalent we prove the following lemma.

Lemma 4.5. Let $T_{X}$ be a lattice given as (1) with $b, c<0$. Then $T_{X}$ is $\mathbb{Z}$-equivalent to $T_{X}^{\prime}$ with $a^{\prime}, b^{\prime}, c^{\prime}<0$.

Proof. Assume that $a \geq 0$ (If $a<0$, there is nothing to prove). Let $A=\left(\begin{array}{lll}0 & 0 & 1 \\ 1 & 0 & 0 \\ 0 & 1 & 0\end{array}\right)$. Since $A \in S L_{3}(\mathbb{Z})$, the matrix $A T_{X} A^{\text {tr }}$ is $\mathbb{Z}$-equivalent to $T_{X}$. Now $T_{X}^{\prime \prime}=$ $A T_{X} A^{t r}=\left(\begin{array}{ccc}2 a^{\prime \prime} & d^{\prime \prime} & e^{\prime \prime} \\ d^{\prime \prime} & 2 b^{\prime \prime} & f^{\prime \prime} \\ e^{\prime \prime} & f^{\prime \prime} & 2 c^{\prime \prime}\end{array}\right)$, where $a^{\prime \prime}=c, b^{\prime \prime}=a, c^{\prime \prime}=b, d^{\prime \prime}=e, e^{\prime \prime}=f$, $f^{\prime \prime}=d$. Then $c^{\prime \prime}<0$. By Lemma 1.6, $T_{X}^{\prime \prime}$ is $\mathbb{Z}$-equivalent to $T_{X}^{\prime}$ with $b^{\prime}, c^{\prime}<0$ and $a^{\prime \prime}=a^{\prime}$. That is, $T_{X}$ is $\mathbb{Z}$-equivalent to $T_{X}^{\prime}$ with $a^{\prime}, b^{\prime}, c^{\prime}<0$.

Now we can assume that $a, b, c$ of Cases 1,2 , and 3 are negative.

Consider the base change by $\left(\begin{array}{lll}0 & 1 & 0 \\ 0 & 0 & 1 \\ 1 & 0 & 0\end{array}\right)$, where $a^{\prime}=b, b^{\prime}=c, c^{\prime}=a, d^{\prime}=f$, $e^{\prime}=d$, and $f^{\prime}=e$. Then Case 2 is reduced to Case 1 . Also the base change by $\left(\begin{array}{lll}0 & 0 & 1 \\ 1 & 0 & 0 \\ 0 & 1 & 0\end{array}\right)$, where $a^{\prime}=c, b^{\prime}=a, c^{\prime}=b, d^{\prime}=e, e^{\prime}=f, f^{\prime}=d$, forces Case 3 to be Case 1 .

Remark 4.6. Whether a K3 surface covers an Enriques surface or not certainly does not depend on the choice of a basis for the transcendental lattice. For some choice of such a basis, if the parities of the integers in $T_{X}$ satisfy one of the conditions in Theorem 1.3, then the parities of $T_{X}$ with respect to any other basis will again satisfy one of the, possibly different, conditions of Theorem 1.3. And the same holding for $T_{X}$ satisfying the conditions of Theorem 1.4.

Now we only consider the case in which only $a$ is odd. In this case we do not exactly know whether the K3 surface cover an Enriques surface. However, there is a partial solution using the spinor genus of an indefinite ternary quadratic form. 
Theorem 4.7 (Eichler, [2]). For indefinite forms of dimension of at least 3, a spinor genus contains exactly one integral equivalence class of forms.

Thus if the spinor genus of the remaining case is the same as one case of Theorem 1.3 and Theorem 1.4, then we know whether the K3 surface is a K3 cover of some Enriques surface.

Acknowledgments. This paper is a revision of my M. S. thesis written at KAIST under the direction of Professor Jonghae Keum. I wish to express my gratitude to my advisor, who not only gave me a lot of help on the subject but also encouraged me to publish it even though it has been many years since this work was first done. I also thank the referee for a very careful reading of the paper, and valuable comments which led to a substantial improvement in the exposition.

\section{References}

[1] W. Barth, K. Hulek, C. Peters, and A. Van de Ven, Compact Complex Surfaces, SpringerVerlag, 2004.

[2] M. Eichler, Quadratische formen und orthogonal gruppen, Springer-Verlag, 1952.

[3] J. Keum, Every algebraic Kummer surface is the K3-cover of an Enriques surface, Nagoya Math. J. 118 (1990), 99-110.

[4] V. Nikulin, Integral symmetric bilinear forms and some of their applications, Math. USSR Izvestija 14 (1980), no. 1, 103-167.

[5] A. Sertöz, Which singular K3 surfaces cover an Enriques surface, Proc. Amer. Math. Soc. 133 (2005), no. 1, 43-50.

Department of Mathematics

University of CALifornia

Riverside, CALIFornia 92521, USA

E-mail address: kwlee@math.ucr.edu 